\title{
A Systems Approach for Identifying Resistance Factors to Rice stripe virus
}

\author{
Kangmin Kim, ${ }^{1}$ Daeseok Choi, ${ }^{2}$ Sang-Min Kim, ${ }^{3}$ Do-Yeon Kwak, ${ }^{4}$ Jaemyung Choi, ${ }^{1}$ Seungchul Lee, ${ }^{2}$ \\ Bong-Choon Lee, ${ }^{3}$ Daehee Hwang, ${ }^{2,5}$ and Ildoo Hwang ${ }^{1}$ \\ ${ }^{1}$ Department of Life Sciences and ${ }^{2}$ School of Interdisciplinary Bioscience and Bioengineering, POSTECH, Pohang, Republic of \\ Korea; ${ }^{3}$ National Institute of Crop Science, RDA, Suwon, Republic of Korea; ${ }^{4}$ National Institute of Crop Science, RDA, Milyang, \\ Republic of Korea; ${ }^{5}$ Integrated Biosciences and Biotechnology, POSTECH, Pohang, Republic of Korea
}

Submitted 8 November 2011. Accepted 11 December 2011.

Rice stripe virus (RSV) causes disease that can severely affect the productivity of rice (Oryza sativa). Several RSVresistant cultivars have been developed. However, host factors conferring RSV resistance in these cultivars are still elusive. Here, we present a systems approach for identifying potential rice resistance factors. We developed two near-isogenic lines (NIL), RSV-resistant NIL22 and RSVsusceptible NIL37, and performed gene expression profiling of the two lines in RSV-infected and RSV-uninfected conditions. We identified 237 differentially expressed genes (DEG) between NIL22 and NIL37. By integrating with known quantitative trait loci (QTL), we selected 11 DEG located within the RSV resistance QTL as RSV resistance factor candidates. Furthermore, we identified 417 DEG between RSV-infected and RSV-uninfected conditions. Using an interaction network-based method, we selected 20 DEG highly interacting with the two sets of DEG as RSV resistance factor candidates. Among the 31 candidates, we selected the final set of 21 potential RSV resistance factors whose differential expression was confirmed in the independent samples using real-time reverse-transcription polymerase chain reaction. Finally, we reconstructed a network model delineating potential association of the 21 selected factors with resistance-related processes. In summary, our approach, based on gene expression profiling, revealed potential host resistance factors and a network model describing their relationships with resistance-related processes, which can be further validated in detailed experiments.

Rice stripe virus (RSV), a member of genus Tenuivirus, causes rice stripe disease that can severely affect the productivity of rice. Infection of RSV is commonly mediated by herbivorous insects such as small brown planthoppers (SBPH) (Laodelphax striatellus) that can transovarially maintain the viability of RSV (Falk and Tsai 1998). RSV infection causes the formation of stripes on leaves and necrosis of newly emerged leaf blades, eventually leading to the complete abro-

\section{K. Kim, D. Choi, and S.-M. Kim contributed equally to this work.}

Corresponding authors: D. Hwang; E-mail: dhhwang@postech.ac.kr; Telephone: +82-54-279-2393; Fax: +82-54-279-8409; and I. Hwang; Email: ihwang@ @ostech.ac.kr; Telephone: +82-54-279-2291; Fax: +82-54279-0629; B.-C. Lee; Telephone: +82-31-290-6792; Fax: +82-31-2955410; E-mail: leebc21@korea.kr

* The $\boldsymbol{e}$-Xtra logo stands for "electronic extra" and indicates that five supplementary tables are published online and Figures 1, 2, and 3 appear in color online. gation of rice growth (Takahashi et al. 1991). Several RSVresistant cultivars (e.g., 'Modan', 'Kanto72', and 'Dular') have been developed and shown to include resistance-related quantitative trait loci (QTL) in chromosomes 2, 3, 6, 8, and 11 . Among them, the QTL in chromosome 11, including qSTV$11 \mathrm{~b}, \mathrm{qSTV}-11 \mathrm{c}, S t v-b^{i}, \mathrm{qSTV} 11^{\mathrm{TQ}}$, and qSTV $11^{\mathrm{KAS}}$, and a QTL from Kanto72, are tightly linked to RSV resistance (HayanoSaito et al. 1998; Hayano-Saito et al. 2000; Maeda et al. 2006; Washio et al. 1968; Wu et al. 2009; Wu et al. 2011; Zhang et al. 2011). However, RSV resistance-related genes within the QTL still remain unknown.

In plant-virus systems, various host resistance factors involved in recessive and dominant resistance-related processes have been identified (Maule et al. 2007; Whitham and Wang 2004). Recessive resistance factors are required for replication, movement, and encapsidation of virus, as well as suppression of host defense (Whitham and Wang 2004), including chaperones, helicases, cytoskeleton components, and translation factors. Dominant resistance factors are involved in resistance $(R)$ gene-mediated responses and small-interfering RNA (siRNA)mediated gene silencing (Lin et al. 2007; Soosaar et al. 2005). For example, NRG1, a coiled-coil nucleotide-binding leucinerich repeat type $R$ gene, was involved in Tobacco mosaic virus (TMV) resistance via the interaction with viral p50 protein (Peart et al. 2005). Also, knockouts of DCL2 that generate siRNAs in antiviral responses showed delayed accumulation of viral siRNAs against Turnip crinkle virus, resulting in increased susceptibility compared with the wild type (Xie et al. 2004). However, only a few rice factors have been shown to play their roles in RSV resistance. For example, knockdown of OsRDR6 required for the biogenesis of siRNAs was recently reported to result in reduced accumulation of RSV-derived siRNA and enhanced susceptibility to RSV (Jiang et al. 2012). Therefore, it is of considerable interest to identify a list of rice factors that can confer resistance to RSV and to understand their collective roles in RSV resistance.

Recently, several gene expression analyses of Pisum sativum, Arabidopsis thaliana, Nicotiana tabacum, and Lycopersicon esculentum infected with various viruses have been performed to investigate molecular responses of hosts to viral infection (Whitham and Wang 2004; Whitham et al. 2006). These studies showed that viral infection induced changes in broad ranges of host cellular processes, including hormone biosynthesis and signaling, induction of heat-shock proteins (HSP), defense, and gene silencing. Recently, gene expression profiling of RSV-infected 'Nipponbare' rice revealed that the expression levels of genes involved in salicylic acid biosynthesis, cell wall synthesis, and energy production were decreased 
after RSV infection, while those of genes involved in jasmonic acid biosynthesis, protein folding, and gene silencing were increased (Satoh et al. 2010). Interestingly, the changes in such cellular processes by RSV infection have been similarly observed in a variety of viruses and hosts (Shimizu et al. 2007; Whitham and Wang 2004; Whitham et al. 2006). These studies have provided us the lists of both genes and cellular processes that are affected by viral infection. However, there has been no systematic approach that can be used to identify RSV resistance factors and a network model describing their association with resistance-related processes based on gene expression data. Here, we present an approach that can systematically identify potential host resistance factors and generate a network model delineating the relationships between the host factors and resistance-associated processes. In this approach, we employed two integration methods to identify potential host resistance factors. The first method involves the integration of differentially expressed genes (DEG) (RSV resistance-related genes) between RSV-resistant and -susceptible lines with the known resistance-related QTL. The DEG that are located within the QTL are likely to be resistance factor candidates. The second method involves the integration of i) DEG (RSV infectionrelated genes) between RSV-uninfected and RSV-infected conditions and ii) the RSV resistance-related genes with their interactome data. The DEG highly interacting with RSV resistance- and infection-related genes are likely to modulate cellular processes associated with these genes. These two sets of DEG can be identified as potential host resistance factors. Network analysis of the potential resistance factors can provide a network model delineating the relationships between the factors and their association with resistance-related processes. Our approach can be applied to host-virus systems in which resistance factors are unknown to identify potential resistance factors and association of the factors with resistance-related processes.

\section{RESULTS AND DISCUSSION}

\section{Development of near-isogenic lines resistant to RSV infection.}

Virus resistance factors can be identified by comparing characteristics of genes and proteins in resistant lines with those in susceptible lines. To this end, we first generated two near-isogenic lines (NIL) that have genetic differences associated with RSV susceptibility. RSV-resistant 'Shinkwang' (subsp. indica) was crossed to RSV-susceptible 'Ilpum' (subsp. japonica) (Fig. 1A) to result in F1 generations which were then backcrossed four times with Ilpum. During each backcross, we selected RSV-resistant individuals and then used them for the next backcrossing. The resulting RSV-resistant and -susceptible descendants $\left(\mathrm{BC}_{4} \mathrm{~F}_{1}\right)$ were then propagated through four more generations $\left(\mathrm{BC}_{4} \mathrm{~F}_{5}\right)$ and designated as NIL22 and NIL37, respectively. Genotyping analyses of NIL using 610 simplesequence repeated (SSR) markers indicate that over $70 \%$ of whole genomes of two NIL lines are identical to those of Ilpum (Supplementary Table S1). The association analysis between genotypic polymorphisms and phenotypic variations for RSV resistance in the NIL showed that RM6897, an SSR marker closely located to $S t v-b^{i}$ on chromosome 11, was most significantly associated with the RSV resistance, consistent to previous findings (Hayano-Saito et al. 1998). In RSV resistance tests, most of the NIL22 lines displayed normal growth at 3 weeks after RSV infection, whereas a majority of NIL37 lines exhibited the necrosis in newly generated leaves and severe growth retardations (Fig. 1B). In closer examination, the unique characteristics of leaf stripes were apparent in NIL37 lines after RSV infection (Fig. 1C). To investigate the nature of the resistance response, we further measured the transcript levels of the RSV coat protein (CP) gene in leaves of six different NIL37 and NIL22 lines collected at 5, 7, and 9 days after RSV infection (Fig. 1D). The transcript of RSV CP genes was
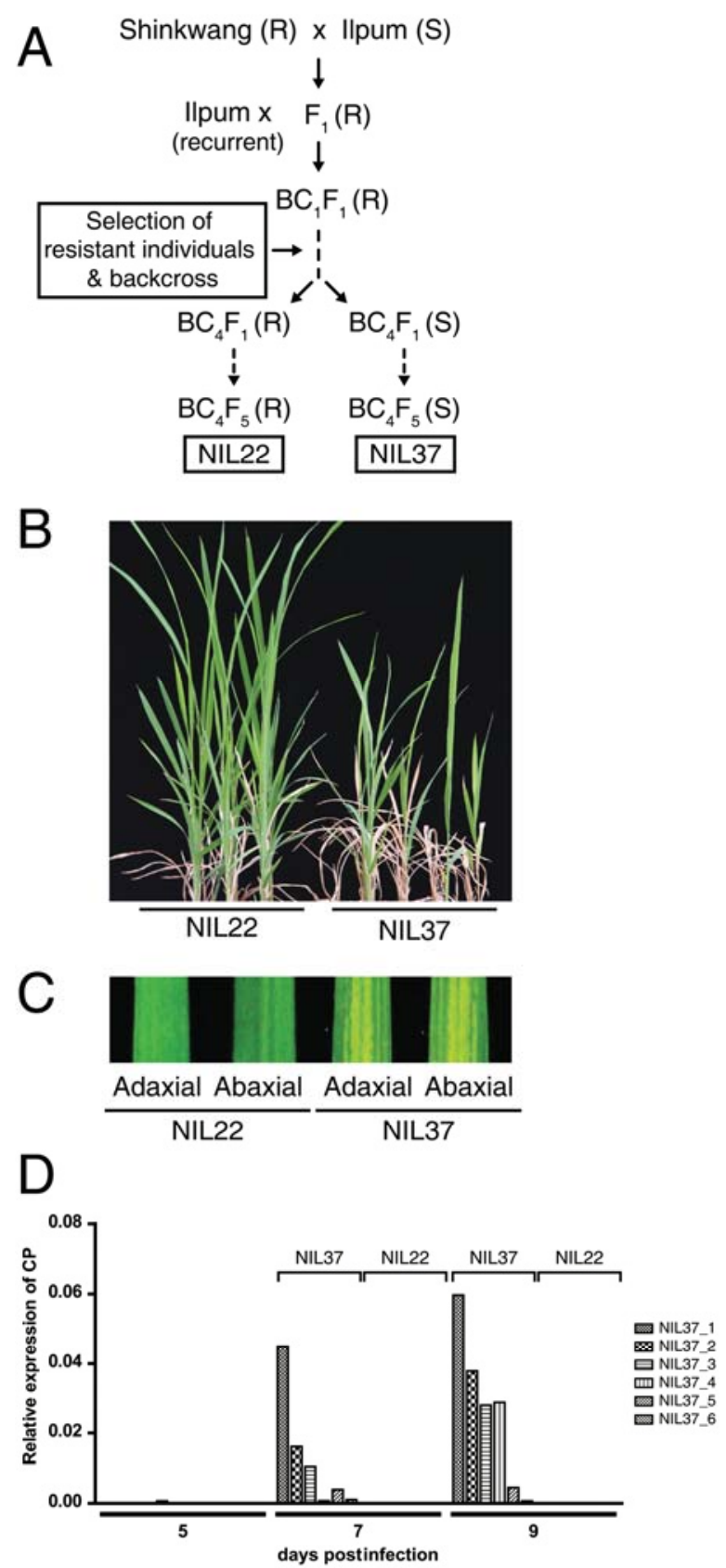

Fig. 1. Development of two independent near-isogenic rice lines (NIL), Rice stripe virus (RSV)-resistant (NIL22) and RSV-susceptible (NIL37). A, Generation of NIL22 (RSV resistant) and NIL37 (RSV susceptible) involving repeated backcrossing of an RSV-resistant 'Shinkwang' with an RSV-susceptible 'Ilpum' and selection of RSV-resistant descendants at each round of crossing. BC, backcross generation; F, filial generation. The subscript numbers denote generations of $\mathrm{BC}$ and $\mathrm{F} ; \mathrm{R}=$ resistant and $\mathrm{S}=$ susceptible. B, Growth retardation and stunting of RSV-infected NIL37 lines at 21 days postinfection (dpi). C, RSV hallmarks of chlorotic stripes on the adaxial and abaxial leaves in RSV-infected NIL37 lines at 21 dpi. D, Transcript levels of RSV coat protein genes in NIL at 5, 7, and 9 dpi. mRNA levels were measured from six different rice seedlings of each NIL. Due to the variability of the expression levels in the infected plants, the measured expression levels were displayed in the individual plants rather than the averaged expression levels. Each datum was normalized by the expression level of LOC_Os03g13170, which encodes ubiquitin fusion protein. 


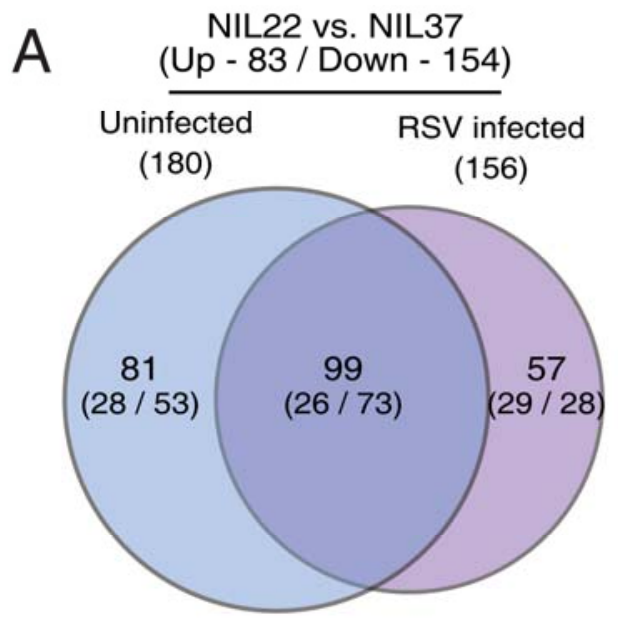

B

\begin{tabular}{ccc}
\hline & GO term & FDR \\
\hline $\begin{array}{c}\text { Down- } \\
\text { regulated }\end{array}$ & cell death & 0.0005 \\
\hline $\begin{array}{c}\text { Up- } \\
\text { regulated }\end{array}$ & secondary metabolic process & 0.0072 \\
gene silencing by miRNA & 0.083 \\
\hline
\end{tabular}
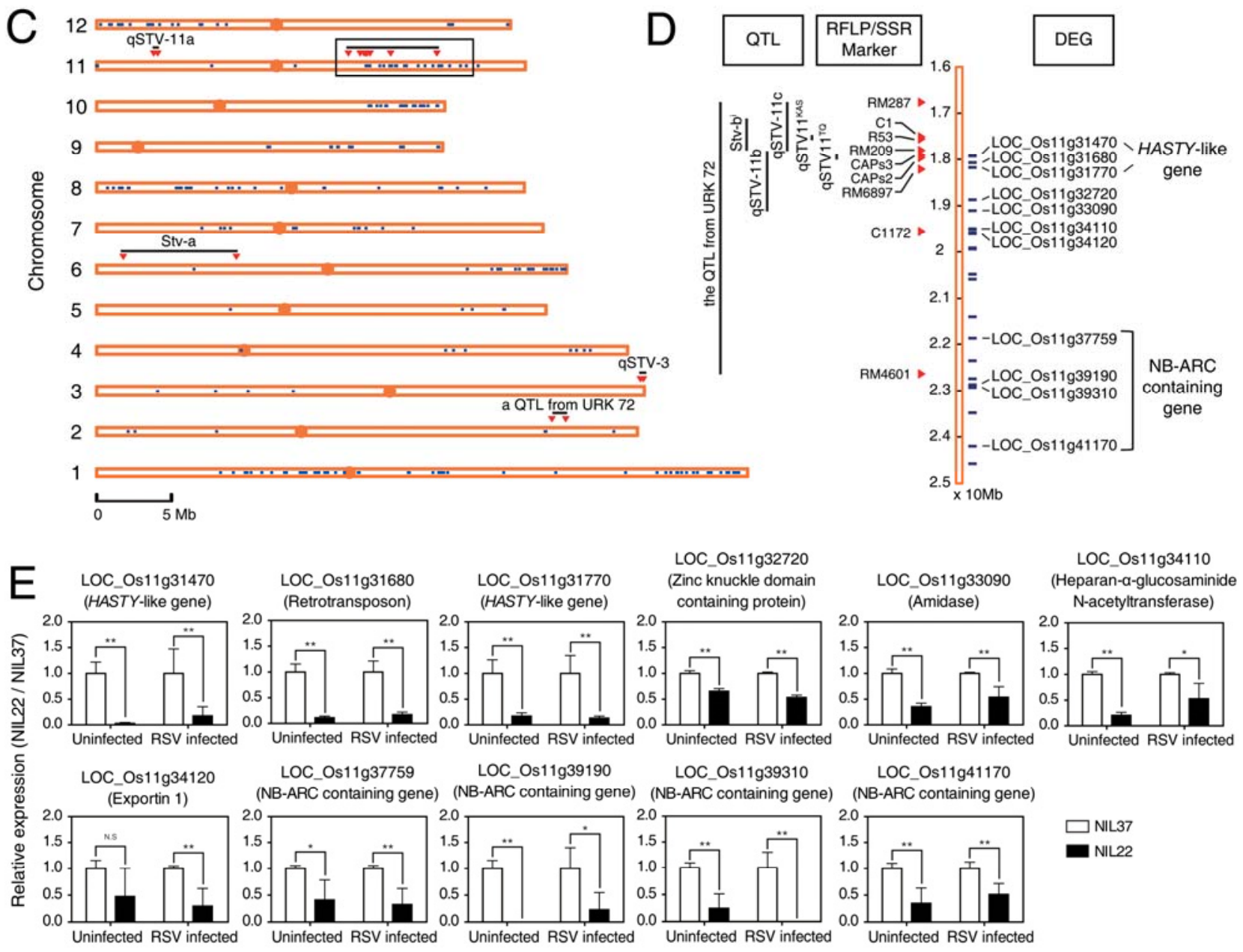

Fig. 2. Rice stripe virus (RSV) resistance-related rice genes and their associated cellular processes. A, Venn diagram showing the relationships of the differentially expressed genes (DEG) between RSV-resistant (NIL22) and RSV-susceptible (NIL37) near-isogenic lines (NIL) in RSV-infected and -uninfected conditions. For each set of the diagram, the numbers of up- and downregulated genes are denoted in parenthesis (up/down, respectively). B, Gene ontology (GO) terms enriched by the RSV resistance-related DEG. FDR = false discovery rate. C, Integration of the DEG with the quantitative trait loci (QTL) from previous genetic association studies. The chromosome plot shows the five QTL, denoted by the black lines, on chromosomes 2 (a QTL from URK72), 3 (qSTV-3), 6 (Stv-A), and 11 (qSTV-11a and the QTL in D [box]). Small squares, DEG; black lines, previously reported RSV resistance QTL; circles, centromeres of the individual chromosomes; triangles, restriction fragment length polymorphism simple-sequence repeat (RFLP/SSR) markers used in the genetic association studies. D, The 11 DEG (horizontal lines) located within or near the QTL (vertical lines) within the box on chromosome 11. Triangles, RFLP/SSR markers. E, Confirmation of the differential expression of the 11 genes in the box on chromosome 11, which were detected by the microarray experiments, by quantitative real-time reverse-transcription polymerase chain reaction. Expression levels of the genes were first normalized by that of LOC_Os03g13170, which encodes ubiquitin fusion protein. For each gene, the normalized expression level was divided by that in NIL37, resulting in an expression level in NIL22 relative to that in NIL37. The relative expression level was separately computed in uninfected and RSV infected conditions. Graphs show the relative expression levels of the 11 genes in uninfected and RSV infected conditions; ** and * indicate $P<0.01$ and 0.05 , respectively (Student's $t$ test). 
mainly detected in NIL37 but not in NIL22 lines. Although they showed a large variability in the individual plants, the $\mathrm{CP}$ expression levels were gradually increased during the course of RSV infection, indicating the increasing titers of RSV in NIL37 during infection. These results suggest that the RSV resistance in NIL22 could be simply due to the reduced titer of RSV. Nonetheless, it is likely that the resistance is due to regulation of RSV resistance-related genes and their collective actions to result in the reduced titer of RSV.

\section{Identification of RSV resistance-related genes.}

To identify DEG involved in RSV resistance, we performed gene expression profiling of both RSV-resistant (NIL22) and RSV-susceptible (NIL37) lines in RSV-infected and RSV-uninfected conditions using Agilent-012106 Rice Oligo Microarray G4138A. We compared the expression levels of each gene between NIL22 and NIL37 in i) RSV-uninfected and ii) RSVinfected conditions, respectively, using a two-tailed $t$ test (Hong et al. 2009; Lee et al. 2010a). From the two comparisons, 180 and 156 DEG, respectively, were identified as the ones with a false discovery rate $($ FDR $)<0.05$ : 99 DEG were shared between RSV-uninfected and RSV-infected conditions while 81 and 57 DEG were specifically identified in RSV-uninfected and RSV-infected conditions, respectively (Fig. 2A). Among the total 237 DEG, 83 genes were upregulated in NIL22 compared with NIL37, whereas 154 genes were downregulated.

To investigate cellular processes or molecular functions associated with RSV resistance-related genes, we then performed functional enrichment analysis of the 237 NIL22-specific DEG (Fig. 2B; Supplementary Table S2) using AgriGO (Du et al. 2010). For the 154 downregulated genes in NIL22, the following three gene ontology (GO) terms were significantly enriched (FDR < 0.1): i) "cell death", including seven genes containing the NB-ARC domain that is highly conserved in plant $R$ genes (Pal et al. 2007; van Ooijen et al. 2008); ii) "secondary metabolic process", including two oxidoreductases, 1-aminocyclopropane-1-carboxylate oxidase (LOC_Os09g27820) and oxidoreductase (LOC_Os10g37880) involved in the biosynthesis of

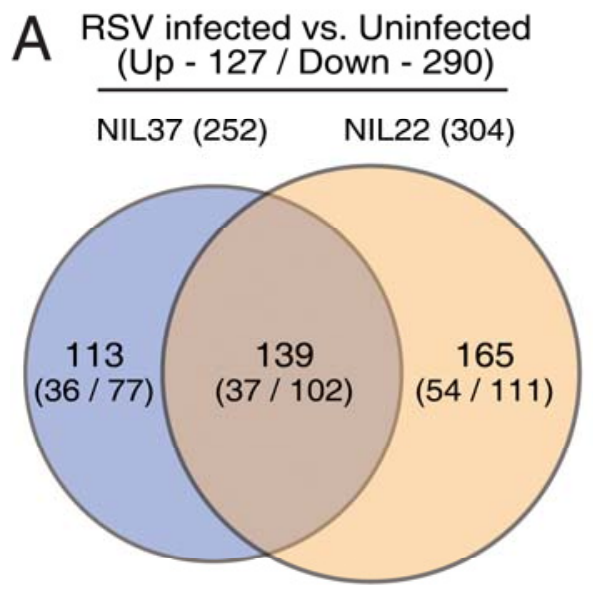

\begin{tabular}{ccc} 
B & & \\
\cline { 2 - 3 } & GO term & FDR \\
\hline $\begin{array}{c}\text { Up- } \\
\text { regulated }\end{array}$ & ATP-dependent helicase activity & 0.00002 \\
& pranslation factor activity & 0.001 \\
$\begin{array}{c}\text { Down- } \\
\text { regulated }\end{array}$ & growth & 0.0063 \\
\hline
\end{tabular}
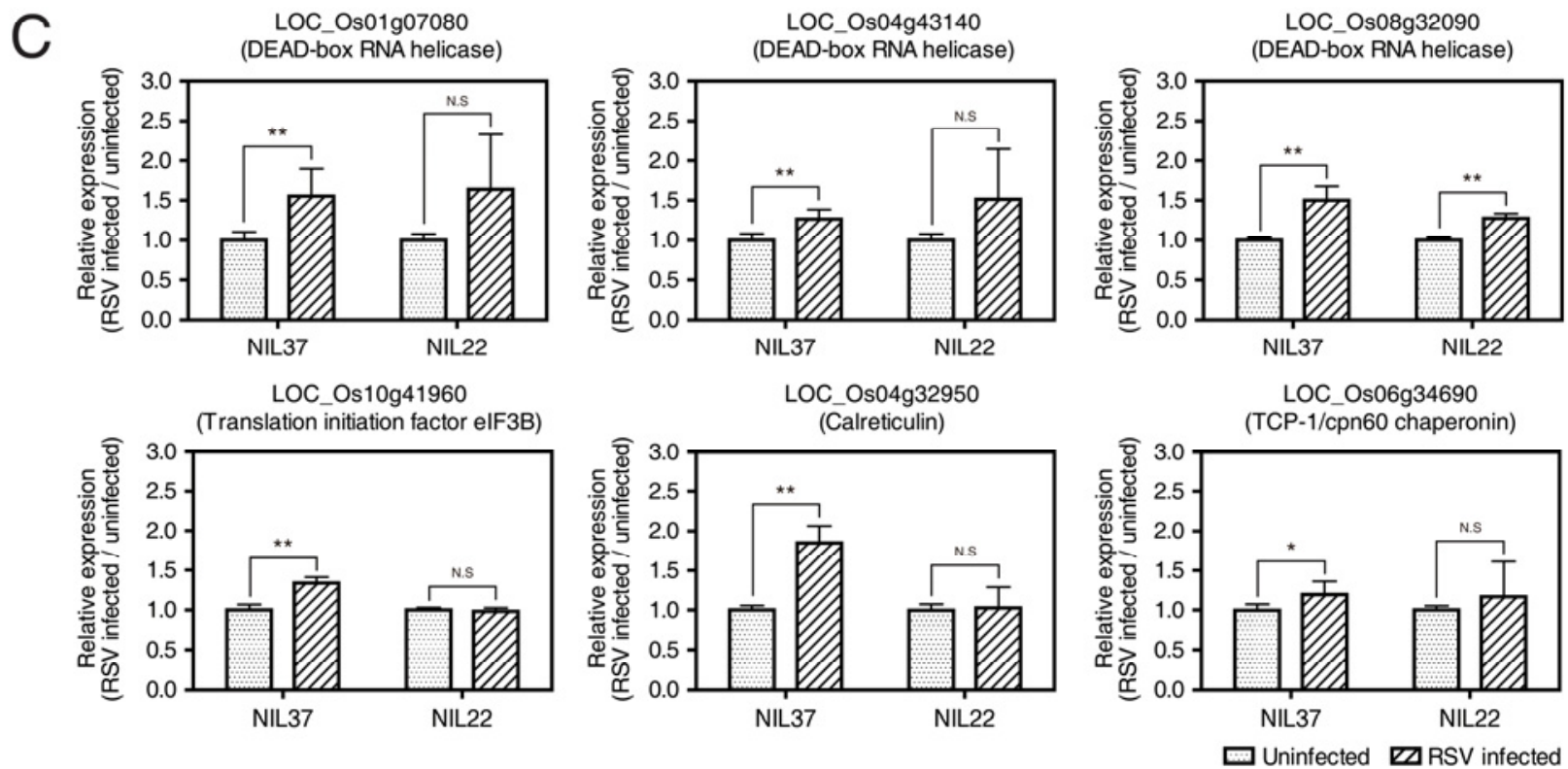

Fig. 3. Rice stripe virus (RSV) infection-related genes and their associated cellular processes in resistant (NIL22) and susceptible (NIL37) lines. A, Venn diagram of the differentially expressed genes (DEG) showing the relationships between RSV-infected and -uninfected conditions. For each set of the diagram, the numbers of up- and downregulated genes are denoted in parenthesis (up/down, respectively). B, Gene ontology (GO) terms enriched by the RSV infection-related DEG. FDR = false discovery rate. C, Confirmation of the differential expression patterns of six genes involved in the enriched GO terms by quantitative real-time reverse-transcription polymerase chain reaction. The expression level was first normalized by that of LOC_Os03g13170. For each gene, the normalized expression level was divided by that in the uninfected condition, resulting in an expression level in the infected condition relative to that in the uninfected condition. The relative expression level was separately computed in NIL22 and NIL37. Graphs show the relative expression levels of the six genes in NIL37 and NIL22; ** and * indicate $P<0.01$ and 0.05 , respectively (Student's $t$ test); N.S. = not significant. 
ethylene that play important roles in viral resistance responses (Fischer and Droge-Laser 2004; Glazebrook 2005; Shin et al. 2004); and iii) "gene silencing by microRNA (miRNA)", including two genes similar to Arabidopsis HASTY involved in nuclear export of pre-miRNAs and generation of mature miRNAs, which are closely associated with growth defects caused by viral infection (Chen et al. 2004; Kasschau et al. 2003). These results suggest their functional association of the identified DEG with RSV resistance. For the 83 upregulated genes in NIL22, "signal transducer activity", including three Ser/Thr protein kinases (STPK) and a receptor-like-kinase, was significantly enriched (FDR $<0.1)$. Several STPK have been also shown to play critical roles in the resistance to TMV (Caplan et al. 2009; Feng et al. 2006; Yafei et al. 2009).

\section{Integration of RSV resistance-related genes with QTL data.}

A number of genetic association studies have shown that several chromosomal regions are strongly associated with RSV resistance (Hayano-Saito et al. 1998, 2000; Maeda et al. 2006; Washio et al. 1968; Wu et al. 2009; Wu et al. 2011; Zhang et al. 2011). To check how the 237 RSV resistance-related DEG are correlated with the 10 QTL identified from previous genetic association studies, we examined chromosomal locations of the DEG (Fig. 2C). Among the five regions including the 10 QTL, the region on chromosome 11, which has been most extensively studied for the last decades, includes the largest number of RSV resistance-related DEG. The other regions, labeled by a QTL from URK72, qSTV-3, Stv-a, and qSTV-11a on chromosomes 2, 3, 6, and 11, respectively, include only one RSV resistance-related DEG on chromosome 6. The following DEG were included in the region on chromosome 11 (Fig. 2D): i) two HASTY-like genes (LOC_Os11g31470 and LOC_Os11g31770), ii) a retrotransposon (RTE, LOC_Os11g31680), iii) LOC_Os11g32720 containing “zinc knuckle domain”, iv) amidase (LOC_Os11g33090), v) heparan- $\alpha$-glucosaminide $N$-acetyltransferase (LOC_Os11g34110), vi) exportin 1 (LOC_ Os11g34120), and vii) an NB-ARC-containing gene (LOC_ Os11g37759). Differential expression of the genes within the cluster was confirmed by the quantitative real-time reverse-transcription polymerase chain reaction (RT-PCR) on independent biological samples (Fig. 2E).

\begin{tabular}{cccc}
\hline Pattern & NIL22/NIL37 & $\begin{array}{c}\text { RSV-infected } \\
\text { / uninfected }\end{array}$ & \#DEG \\
\hline 1 & - & Down & 279 \\
2 & Down & - & 145 \\
3 & - & Up & 122 \\
4 & Up & - & 76 \\
5 & Up & Down & 6 \\
6 & Down & Down & 5 \\
7 & Down & Up & 4 \\
8 & Up & Up & 1 \\
\hline
\end{tabular}

Total : 638

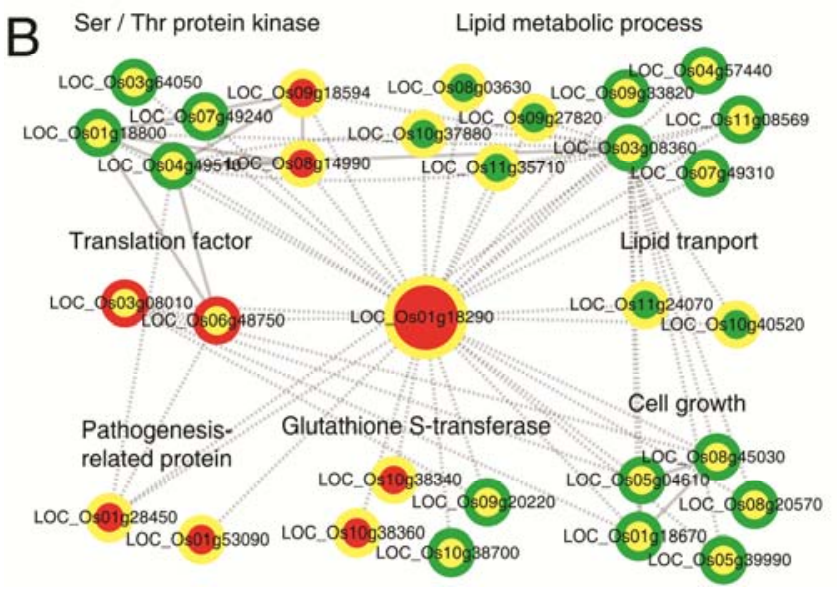

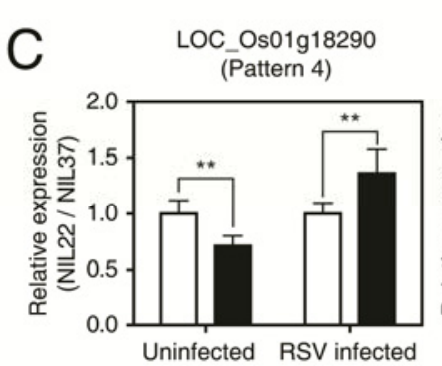
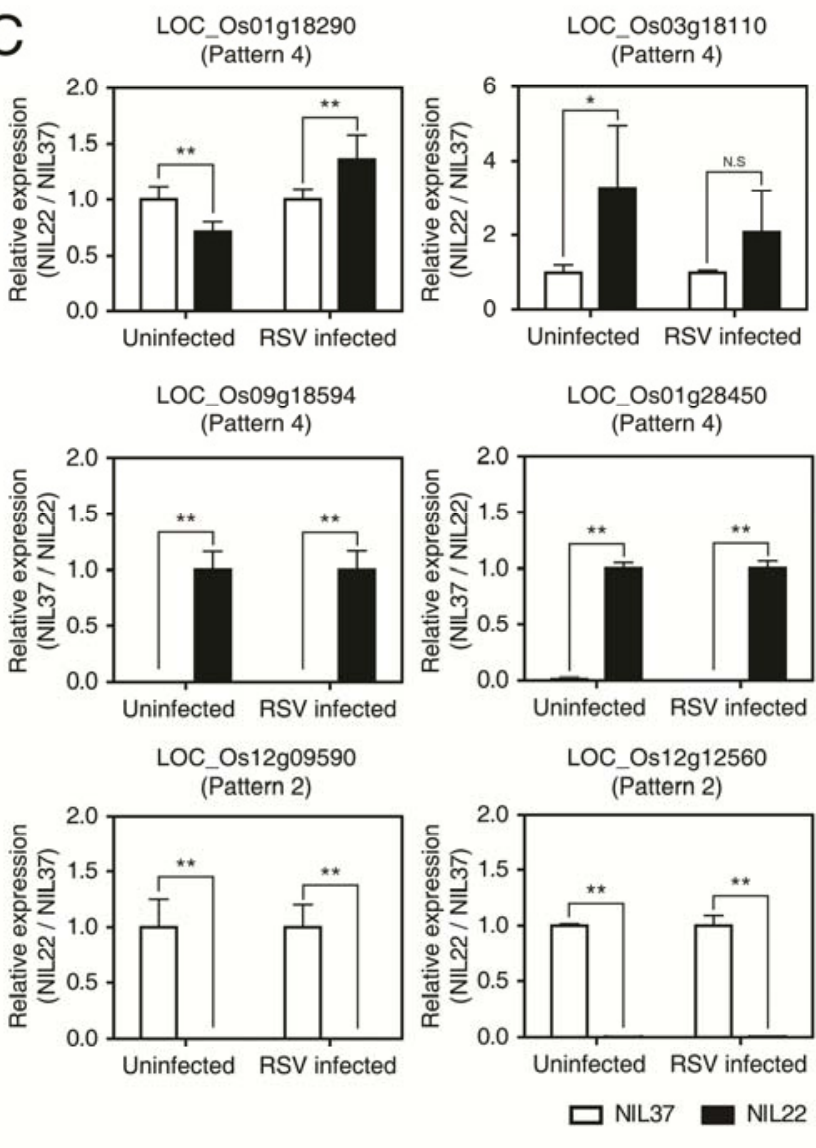

Fig. 4. Identification of potential Rice stripe virus (RSV) resistance factors. A, Distribution of the two sets of the differentially expressed genes (DEG) in the eight groups defined by their differential expression patterns. Up = upregulated in near-isogenic line (NIL)22 lines or RSV-infected conditions; Down = downregulated. B, Network showing the DEG interactors of the top candidate gene (LOC_Os01g18290) and their associated cellular processes. Nodes represent the DEG interactors. Node (center) color represents upregulation (red) and downregulation (green) in the comparison of NIL22 versus NIL37 conditions, and the node boundary (outline) color represents upregulation (red) and downregulation (green) in the comparison of RSV-infected versus -uninfected conditions. Yellow node and node boundary colors represent no significant changes in the corresponding comparisons. Dotted lines, genetic associations including protein-DNA, genetic, and predicted interactions; solid lines, experimentally validated protein-protein interactions. C, Validation of RSV resistance and infection-related differential expression of the selected factor candidates by quantitative real-time reverse-transcription polymerase chain reaction. The expression level was normalized by that of LOC_Os03g13170. For LOC_Os01g18290, LOC_Os03g18110, LOC_Os12g09590, and LOC_Os12g12560, the normalized expression level was divided by that in NIL37, resulting in an expression level in NIL22 relative to that in NIL37. For LOC_Os09g18594 and LOC_Os01g28450, the normalized expression level was divided by that in NIL22 due to their residual levels in NIL37. The relative expression level was separately computed in RSV-uninfected and -infected conditions. Graphs show the relative expression levels in RSV-uninfected and -infected conditions; ** and * indicate $P<0.01$ and 0.05 , respectively (Student's $t$ test); N.S. = not significant. 
Interestingly, several DEG in the cluster on chromosome 11 have been shown to be associated with resistance to pathogens. For example, knockout of an RTE, AtCOPIA4, decreased the resistance to Hyaloperonospora parasitica in Arabidopsis (Wang and Warren 2010). The transient expression of an RTE, Tnt 1 , in tobacco reduced the expression of its neighboring resistance-related gene, RPP4, by forming double-stranded RNA that could trigger RNA silencing (Hernandez-Pinzon et al. 2009). A putative heparan- $\alpha$-glucosaminide $N$-acetyltransferase (LOC_Os11g34110), which is involved in catabolism of heparan sulfate that in invertebrates and acts as the receptor for Herpes Simplex virus and Adeno-associated virus (Spear et al. 1992; Summerford and Samulski 1998), can affect virus resistance by modulating recognition of viruses. Exportin 5, a mammalian ortholog of HASTY, transports virus-associated (VA) RNAs, in addition to pre-miRNAs, to cytoplasm. VA RNAs were shown to be required for virus viability by inhibiting interferon-induced antiviral defense system (Gwizdek et al. 2003; Kitajewski et al. 1986). These observations suggest potential roles of the resistance-related DEG in the cluster in RSV resistance.

Unexpectedly, four NB-ARC genes in the gene cluster were downregulated in NIL22, compared with NIL37 lines, which seems to be inconsistent with previous findings that the activation of $R$ genes should enhance viral resistance (Soosaar et al. 2005). The unexpected downregulation of these NB-ARC genes could be simply due to less functional needs for the induction of these genes with the low RSV titer in NIL22 lines (Fig. 1D). However, considering the residual RSV titer, it is not still clear why only the particular $R$ genes in the cluster of QTL on chromosome 11 showed downregulation in NIL22 lines and what caused their downregulation. Therefore, it is uncertain whether the downregulation of these genes could be caused by passive or active mechanisms affecting their gene expression levels.

Taken together, the results from the integrative analysis suggest that these genes in the gene cluster on chromosome 11 might have potential associations with viral resistance in rice, considering that this gene cluster sustained the genome of RSVresistant Shinkwang and their orthologs are associated with resistance against pathogens in various organisms.

\section{Identification of rice genes affected by RSV infection.}

Virus resistance is mediated by the alteration of the gene expression caused by viral infection (Whitham et al. 2006). To identify such genes affected by the RSV infection, we compared expression levels between RSV-infected and RSV-uninfected conditions in both NIL22 and NIL37. From the two comparisons, we identified 417 DEG using the same twotailed $t$ test. Among them, 139 DEG exhibited shared differential expression patterns in NIL22 and NIL37 lines, whereas 113 and 165 DEG showed specific patterns in NIL22 and

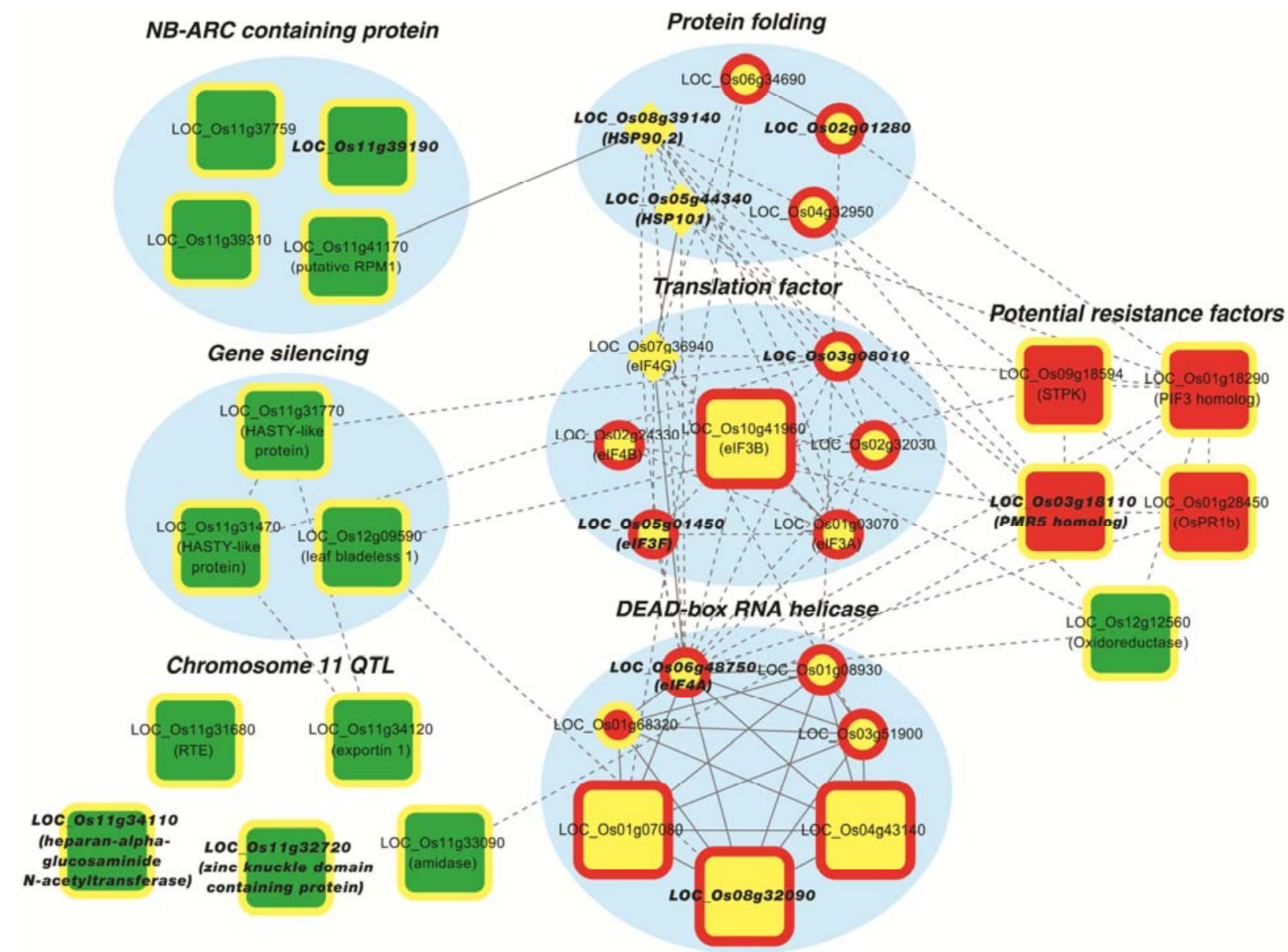

Fig. 5. Hypothetical Rice stripe virus (RSV) resistance network. The network shows several functional modules indicated by the blue background. Each module includes the nodes with the same gene ontology (GO) term and is labeled by the corresponding GO term. Nodes represent the 21 selected factors (rounded rectangles), the RSV resistance and infection-related differentially expressed genes (DEG) (circles) with the same GO term, and the known resistance factors connecting the selected factors (diamonds). Node (center) color represent upregulation (red) and downregulation (green) in the comparison of NIL22 versus NIL37 conditions, and the node boundary (outline) color represents upregulation (red) and downregulation (green) in the comparison of RSV-infected versus -uninfected conditions. Yellow node and node boundary colors represent no significant changes in the corresponding comparisons. Edges represent protein-protein interactions (solid lines) and genetic associations, including genetic and predicted interactions (dotted lines). Bold node labels indicate the genes whose differential expression was also previously detected after RSV infection (Satoh et al. 2010). 
NIL37, respectively (Fig. 3A). Among the 417 DEG, 127 genes are upregulated in either NIL22 or NIL37 lines by RSV infections while 290 genes are downregulated. These DEG significantly overlapped (FDR $<10^{-4}$ ) with those previously reported (Satoh et al. 2010) (Supplementary Table S3). Using these DEG, we then performed functional enrichment analysis to identify cellular processes and molecular functions represented by them (Fig. 3B). The 127 upregulated genes after RSV infection mainly had the following functions: i) "ATP-dependent helicase activity", including DEAD-box RNA helicases; ii) "translation factors activity", including three translation initiation and two elongation factors; and iii) "protein folding", including six chaperones. The 290 downregulated genes after RSV infection were mainly involved in "growth", including three expansins, and "lipid metabolic process", including four lipases.

Several upregulated genes have been previously shown to be involved in virus replication or recessive resistance in various plant-virus systems (Whitham and Wang 2004; Whitham et al. 2006). An Arabidopsis DEAD-box RNA helicase, AtRH8, interacts with the viral genome-linked protein, VPg, of Potyvirus spp. and is required for the accumulation of the viral RNA of Potyvirus spp. (Huang et al. 2010). Also, the translation initiation factors (e.g., eIF4E and eIF(iso)4E) are required for Potyvirus spp. replication and cell-to-cell trafficking in Arabidopsis, tobacco, and melon (Duprat et al. 2002; Gao et al. 2004; Nieto et al. 2006), and eIF(iso)4E knockout mutants showed enhanced resistance to Turnip mosaic virus (Lellis et al. 2002). HSP70 and its cochaperone CPIP were shown to regulate the multiplication of Potato virus A by destabilizing its $\mathrm{CP}$, which could inhibit viral translation and replication in tobacco (Hafren et al. 2010).

Based on their roles as recessive resistance factors, it is expected that these genes are suppressed for hosts to acquire virus resistance. Our data showed that these genes were upregulated in RSV-susceptible NIL37 lines but that expression levels of most of these genes were not significantly changed in RSVresistant NIL22 lines (Fig. 3C). No change in their expression levels in NIL22 could be due to less functional need for the induction of these genes with the low RSV titer in NIL22 lines. On the other hand, the upregulation of these genes has been previously observed in another RSV-susceptible line, Wuyun3, after RSV infection (GSE11025). Similarly, protein abundance of translation factors and chaperones, including CPN60, was found to be increased in virus-susceptible rice cells infected with Rice yellow mottle virus (Ventelon-Debout et al. 2004). Moreover, HSP, including HSP17.6A, HSP83, and HSP70, were shown to be induced in Arabidopsis in response to accumulation of viral protein during the infection of diverse RNA viruses (Aparicio et al. 2005; Whitham et al. 2003, 2006).

Notably, these genes were shown to be actively involved in or required for pathogen resistance. For example, DEAD-box RNA helicases are involved in unwinding siRNAs and to affect the silencing of host and viral genes during viral infection in plants and Drosophila spp. (Linder and Owttrim 2009; Soosaar et al. 2005; Ulvila et al. 2010). A chaperone, HSP90, was also shown to be required for $R$-gene-mediated resistance involving Rx1 and N, as well as RPM1 and RPS2 in tobacco, potato, and Arabidopsis (Hubert et al. 2003; Liu et al. 2004; Lu et al. 2003; Takahashi et al. 2003). Also, transgenic Arabidopsis-overexpressing OsBIRH1, a rice DEAD-box helicase, showed enhanced resistance to Pseudomonas syringae pv. tomato and Alternaria brassicicola (Li et al. 2008).

Although upregulation of these genes still remains to be explained, they collectively appear to interact with virus resistance factors. For example, the DEAD-box helicases form a translation initiation complex (Robaglia and Caranta 2006) by interacting with translation initiation factors, which can be recruited to viral RNAs together with HSP (e.g., HSP101) (Gallie 2002; Noueiry et al. 2000). HSP90 also forms complexes with $R$ genes in Arabidopsis to establish $R$-gene-mediated resistance. Taken together, the results suggest a hypothesis that a certain set of DEAD-box helicases, chaperones, and translation factor can collectively modulate both viral propagation and plant resistance through their interactions with resistance-related molecules. This hypothesis prompted identification of potential resistance factors among these genes affected by RSV infection based on their interactions with both resistance- and infection-related DEG below.

\section{Interaction network-based identification of potential RSV resistance factor candidates.}

Among the RSV resistance-related genes (237 DEG) (Fig. 2A) and the genes affected by RSV infection (417 DEG) (Fig. $3 \mathrm{~A}$ ), which genes can affect most significantly the RSV resistance- or infection-associated processes? Based on the results from the integration of the RSV resistance-related genes with the QTL associated with RSV resistance, we first selected a set of RSV resistance factor candidates as the 11 genes within the cluster on chromosome 11 (Fig. 2D). In parallel, we also employed an interaction network-based method to search for potential RSV resistance factors. We first categorized the two sets of DEG (a total of $638 \mathrm{DEG)}$ into eight groups based on their differential expression patterns (DEP) (Fig. 4A). A majority of DEG (97.5\% of DEG) showed the four major DEP (patterns 1 to 4) unique to either RSV resistance or infection, whereas only small numbers of DEG showed the shared DEP (patterns 5 to 7). In this method, we hypothesized that a factor interacting with a larger number of DEG (e.g., a hub regulator) can significantly affect the RSV resistance- or infection-associated processes in which the DEG are involved because each interaction has a functional link between the interactors (e.g., protein-protein interactions such as kinase-substrate interactions, transcription factor (TF)-target interactions, or genetic associations). Based on this hypothesis, using the interaction network-based method, we selected 20 potential host resistance factors with statistically significant numbers of interactors $($ FDR $<0.05)$ as another set of potential RSV resistance factors (Table 1). Among the 31 potential resistance factor candidates, seven genes overlapped with the RSV infection-related genes previously reported (Satoh et al. 2010): among the first set of 11 factor candidates, an NB-ARC containing protein (LOC_Os11g39190), heparan- $\alpha$-glucosaminide $N$-acetyltransferase (LOC_Os11g34110), zinc knuckle domain containing protein (LOC_Os11g32720), and PMR5 homolog (LOC_Os03g18110); and among the second set of 20 factor candidates, two DEAD-box RNA helicases (LOC_Os06g48750 and LOC_Os08g32090) and a translation initiation factor (LOC_Os03g08010).

\section{Validation of differential expression of resistance factor candidates.}

Using real-time RT-PCR, we then tested differential expression of the $31 \mathrm{RSV}$ resistance factor candidates in the independent NIL22 and NIL37 samples under RSV-infected and RSV-uninfected conditions. Among the 31 candidates, we selected the 21 potential RSV resistance factors whose differential expression was confirmed in the independent samples (11 factors in Figure 2E and 10 factors in Figures 3C and 4C). The 21 potential RSV resistance factors belong to the RSV resistance- and infection-related cellular processes (Figs. 2B and 3B), suggesting the possibility that these processes can act together to acquire RSV resistance. The top candidate (LOC_ Os01g18290), a helix-loop-helix DNA-binding protein homologous to Arabidopsis PIF3, which is involved in the light-signal- 
ing pathway in Arabidopsis, interacts with 187 DEG, including STPK, translation factor, cell growth, glutathione $S$-transferases, pathogenesis-related proteins, and lipid metabolic/transport processes (Fig. 4B). The network suggests that the PIF3 homolog may modulate RSV resistance through the interactions with a broad spectrum of RSV resistance-related cellular processes.

Most of the selected factors have been reported to be associated with pathogen resistance in rice and other plants. As aforementioned, DEAD-box RNA helicases (LOC_Os04g43140, LOC_Os08g32090, and LOC_Os01g07080) and translation factor (LOC_Os10g41960) are associated with virus resistance as well as viral replication and propagation. Moreover, LOC Os12g09590 (leaf bladeless 1) is homologous to SGS3 that inhibits the virus replication and propagation in hosts through viral gene silencing (Glick et al. 2008; Mourrain et al. 2000). LOC_Os03g18110 is also homologous to Arabidopsis PMR5, whose mutations resulted in powdery mildew resistance (Vogel et al. 2004). LOC_Os01g28450, OsPR1b, was previously reported to be increased in expression level after infections with pathogens, and may act as an anti-fungal agent (Agrawal et al. 2001; Mitsuhara et al. 2008; Van Loon and Van Strien 1999). LOC_Os09g18594, a leucine-rich receptor-like protein kinase, can play various roles in plant innate immunity (Afzal et al. 2008), thus modulating virus resistance. All the associations of the selected factors with virus resistance support the hypothesis that they can act as potential important regulators of RSV resistance.

\section{Reconstruction of a hypothetical RSV resistance network.}

To understand how these selected factors (Fig. 2D; Table 1) can be collectively linked to the RSV resistance- and infectionrelated cellular processes (Figs. 2B and 3B), we reconstructed a hypothetical RSV resistance network (Fig. 5) using the 21 selected factors and their interacting DEG involved in the RSV resistance- and infection-related processes. The network shows several functional modules: gene silencing, NB-ARC-containing protein, protein folding, translation factor, and DEAD-box RNA helicase modules. The network further revealed the interactions among the selected factors within and between the modules. First, the selected factors in DEAD-box RNA helicase and translation factor modules closely interact with the molecules within the modules, reflecting that they are likely to act collectively in regard to RSV resistance. Second, the molecules in gene silencing, protein folding, and DEAD-box RNA helicase modules are closely linked via the nodes in the translation factor module. Moreover, HSP90.2 (LOC_Os08g39140), an interactor of RPM1, and HSP101 (LOC_Os05g44340), a component of eIF4G-viral RNA complex, interact with a number of the selected factors and RSV resistance- and infectionrelated DEG in the network (Gallie 2002; Hubert et al. 2003). These observations suggest that the translation factor and the HSP may play roles in RSV resistance- and infection-related processes as potential functional hubs. These hub-like proteins are densely linked to the molecules, including i) HASTY-like proteins (gene-silencing module) and ii) LOC_Os01g07080, LOC_Os04g43140, and LOC_Os08g32090 (DEAD-box RNA helicase module), as well as iii) several potential resistance factors (LOC_Os09g18594, LOC_Os01g18290, LOC_Os03g18110, LOC_Os01g28450, and LOC_Os12g12560). The dense links indicate that these molecules could have important roles in RSV resistance through their interactions with the hub-like proteins. Finally, the factors in the NB-ARC-containing protein module have few interactions reported, indicating that the understanding of the interactions between $R$ genes and the molecules in other modules is currently limited. In summary, the network model suggests that the RSV resistance involves close interactions between the molecules, reflecting the possibility of their collective actions in the context of RSV resistance, though the network shows a limited view of interactions due to the incomplete interactome data.

\section{Conclusions.}

We introduced a systems approach for identifying potential viral resistance factors and a network model delineating their potential association with resistance-related processes. This approach involves the identification of viral resistance-related genes from global studies, selection of potential viral resistance factors through the integration of the resistance-related genes,

Table 1. Potential Rice stripe virus (RSV) resistance factors identified by the interaction network-based method

\begin{tabular}{|c|c|c|c|c|}
\hline Description & Gene ID & Pattern $^{\text {a }}$ & $\begin{array}{l}\text { Number of } \\
\text { interactors }^{b}\end{array}$ & FDR $^{\mathbf{c}}$ \\
\hline Helix-loop-helix DNA-binding domain containing protein (PIF3 homolog) & LOC_Os01g18290d & 4 & 187 & 0.001 \\
\hline PMR5 homolog & LOC_Os03g18110 d & 4 & 103 & 0.005 \\
\hline NAP domain containing protein & LOC_Os05g46230 & 3 & 69 & 0.008 \\
\hline Leafbladeless1 (SGS3 homolog) & LOC_Os $12 \mathrm{~g} 09590^{\mathrm{d}}$ & 2 & 57 & 0.009 \\
\hline ATP-dependent Clp protease subunit clpA homolog & LOC_Os12g12850 & 3 & 25 & 0.022 \\
\hline Ribosome biogenesis regulatory protein & LOC_Os04g58830 & 3 & 23 & 0.027 \\
\hline Protein kinase domain containing protein & LOC_Os09g18594 & 4 & 19 & 0.034 \\
\hline Pathogenesis-related protein OsPR1b & LOC_Os01g28450 d & 4 & 18 & 0.037 \\
\hline NADP-dependent oxidoreductase & LOC_Os12g12560 & 2 & 16 & 0.045 \\
\hline Plasminogen activator inhibitor 1 RNA-binding protein & LOC_Os01g52390 & 3 & 16 & 0.045 \\
\hline \multirow[t]{6}{*}{ DEAD-box ATP-dependent RNA helicases } & LOC_Os06g48750 & 3 & 45 & 0.010 \\
\hline & LOC_Os04g43140 & 3 & 29 & 0.018 \\
\hline & LOC_Os08g32090 d,e & 3 & 21 & 0.029 \\
\hline & LOC_Os03g51900 & 3 & 20 & 0.032 \\
\hline & LOC_Os01g07080 d & 3 & 17 & 0.041 \\
\hline & LOC_Os01g68320 & 4 & 22 & 0.028 \\
\hline \multirow[t]{4}{*}{ Translation initiation/elongation factors } & LOC_Os03g08010 & 3 & 36 & 0.013 \\
\hline & LOC_Os02g32030 & 3 & 26 & 0.020 \\
\hline & LOC_Os10g41960 d & 3 & 19 & 0.034 \\
\hline & LOC_Os01g03070 & 3 & 16 & 0.045 \\
\hline
\end{tabular}

${ }^{a}$ Differential expression pattern of potential resistance factors; $2=$ downregulated in lines of RSV-resistant near-isogenic line (NIL)22, $3=$ upregulated in RSV-infected conditions, and $4=$ upregulated in NIL22 lines.

${ }^{\mathrm{b}}$ Number of interactors among the resistance- and infection-related differentially expressed genes (DEG).

${ }^{c}$ Significance of having the number of DEG interactors (false discovery rate [FDR]).

${ }^{\mathrm{d}}$ Genes whose expression was confirmed by real-time reverse-transcription polymerase chain reaction in the independent samples.

${ }^{\mathrm{e}}$ Gene whose expression was previously reported to be significantly changed in RSV infected conditions (Satoh et al. 2010). 
the QTL, and the interactome data; and reconstruction of a network model describing the relationships between the selected factors and their associated cellular processes. In this study, we demonstrated the utility of this systems approach by applying it to RSV resistance to identify 21 potential RSV resistance factors. A number of the identified potential resistance factors appear to be associated with viral resistance and infection based on previous literatures, supporting the validity of the proposed approach. The network model describing the relationships between the 21 factors and resistance-related processes further suggested a particular set of potential rice resistance factors (two HASTY-like proteins, three DEAD-box RNA helicases, and five potential resistance factors) having relatively dense links to hublike translation factor and HSP. Their potential roles in RSV resistance can be tested in detailed functional studies. In conclusion, this proposed approach is applicable to other plant-virus systems in which resistance factors and the networks associated with resistance are unknown. Therefore, the approach can offer new opportunities for enhancing our understanding of virus resistance factors and their associated networks.

\section{MATERIALS AND METHODS}

\section{Plant materials and RNA extraction.}

For microarray experiments, young seedlings of NIL22 (RSVresistant) and NIL37 (RSV-susceptible) lines (Fig. 1) that have been grown in pots in a greenhouse for 7 days were exposed to healthy and RSV-viruliferous SBPH (Laodelphax striatellus). After 2 days, SBPH were removed, and the seedlings were further incubated in the same greenhouse, separated by different containers. After 7 days postinfection (dpi), leaf tissues of individual seedlings were frozen in liquid $\mathrm{N}_{2}$ and stored separately at $-80^{\circ} \mathrm{C}$. Virus-infected samples were selected by RSV CP targeted enzyme-linked immunosorbent assay and RT-PCR analyses using the remaining leaves of RSV-infected seedlings after another 7 days. Two biological replicates for microarray experiments were prepared in the same growth and infection conditions. We focused on the early responses to the RSV infection because we hypothesized that the interactions between rice and RSV factors, associated with RSV resistance, were most clearly observed during the early infection period.

\section{Kinetics of RSV propagation in NIL22 and NIL37.}

From each NIL, the mRNAs were isolated from the leaf tissues of six seedlings at 5, 7, and $9 \mathrm{dpi}$. The expression level of the RSV CP gene was measured by real-time RT-PCR using RSV CP gene-specific primers. The data were normalized using the expression level of the gene-encoding ubiquitin fusion protein (LOC_Os03g13170) that showed a constant expression level in NIL37 and NIL22 in uninfected and RSV-infected conditions.

\section{Gene expression analysis.}

Total RNA was isolated from two biological replicates of leaf tissues of RSV-infected and RSV-uninfected NIL using Trizol Reagent (Invitrogen, San Diego, CA, U.S.A.). The integrity of RNA was measured using Bioanalyzer (Agilent Technologies, Santa Clara, CA, U.S.A.). RNA with a high RNA integrity number $(>9.2)$ was used for the microarray experiment. The following steps were performed according to the Agilent protocols. Briefly, cyanine 3 (Cy3)- or cyanine 5 (Cy5)-labeled cRNAs were synthesized from $500 \mathrm{ng}$ of total RNAs by using a Low RNA Input Linear Amplification Kit (Agilent Technologies). Hybridization solution containing $1 \mathrm{mg}$ each of $\mathrm{Cy} 3-$ and Cy-5-labeled cRNA was prepared with the In situ Hybridization Kit Plus (Agilent Technologies). The labeled cRNAs were hybridized to the Rice Oligo microarray G4138A (Agilent
Technologies) by incubating at $60^{\circ} \mathrm{C}$ for $17 \mathrm{~h}$. The array slides were washed twice (first with $6 \times \mathrm{SSC}[1 \times \mathrm{SSC}$ is $0.15 \mathrm{M} \mathrm{NaCl}$ plus $0.015 \mathrm{M}$ sodium citrate] $+0.005 \%$ TritonX-102 for 10 min at $23^{\circ} \mathrm{C}$ and second with $0.1 \times \mathrm{SSC}+0.005 \%$ TritonX-102 for $5 \mathrm{~min}$ at $4^{\circ} \mathrm{C}$ ) and then dried. Image files of the fluorescent signal on the slides were produced with the DNA microarray scanner (Agilent Technologies). Signal intensities were measured from the image files by Feature Extraction (version 7.5.1; Agilent Technologies). The microarray data used in this study is available at the National Center for Biotechnology Information GEO database (GSE 6126).

\section{Normalization of gene expression data.}

Median signal intensities of $\mathrm{Cy} 3$ and $\mathrm{Cy} 5$ were converted to $\log _{2}$ median intensities. We then normalized the $\log _{2}$ intensities using the quantile normalization method (Bolstad et al. 2003). A gene was considered "expressed" if the probe intensity of the gene was larger than a cutoff intensity at least at one condition. The cutoff intensity (in this study, intensity $=6.95$ ) was determined by the mixture modeling using two Gaussian distributions, one for the "absent" probes and the other for the "present" (expressed) probes (Hong et al. 2009; Lee et al. 2010a).

\section{Identification of DEG.}

To identify DEG from the four comparisons (RSV-uninfected NIL22 versus NIL37, RSV-infected NIL22 versus NIL37, RSVuninfected versus RSV-infected NIL22, and RSV-uninfected versus RSV-infected NIL37), the following statistical hypothesis test method was applied. In each comparison, i) $t$ statistics were calculated from the two-tailed $t$ test for the two sample groups with unequal variance; ii) an empirical distribution of the $t$ statistics was computed using the $t$ statistic values calculated from hundreds of random permutations of the eight samples in all the conditions; and iii) FDR were computed using the empirical distribution based on Storey's method (Nurieva et al. 2008; Storey and Tibshirani 2003). The DEG were identified as the ones with FDR $\leq 0.05$. We then further removed false positives i) whose fold changes were between -0.58 and 0.58 at the $\log _{2}$ scale and ii) whose gap sizes were less than 0.4 at the $\log _{2}$ scale. The gap size was defined by the difference between the minimum intensity in group A with the larger mean intensity and the maximum intensity in group B with the smaller mean intensity. The cutoff values were found as $95 \%$ percentiles of the fold changes and the gap sizes obtained from the random permutation experiments at the two-tailed settings.

\section{Functional enrichment analysis of DEG.}

Using AgriGO Analysis Toolkit and Database for Agricultural Community (Du et al. 2010), we identified the GO biological processes or molecular functions significantly enriched (FDR < 0.1) by the DEG (e.g., for the RSV resistance-related DEG, a union of DEG from RSV-uninfected NIL22 versus NIL37 and RSV-infected NIL22 versus NIL37). We used the FDR values provided from Fisher's exact test in AgriGO.

\section{Rice interactome database.}

To select potential resistance factors based on the interactome data and also to reconstruct a network model, we developed a rice interactome database by integrating the following resources: i) AtORFeome2.0 (Vidal et al. 2011); ii) PPIN-1 (Mukhtar et al. 2011); iii) Arabidopsis-curated protein-protein interactions from The Arabidopsis Information Resource (TAIR); iv) TAIR Interactome 2.0, including predicted protein-protein interactions based on orthologous interactions in other species (Geisler-Lee et al. 2007); v) multinetwork (Gutierrez et al. 2007); vi) Aranet (Lee et al. 2010b); vii) curated protein-protein interactions from previous literatures, which were detected by conventional 
assays (e.g., yeast two-hybrid assays, co-immunoprecipitations, bimolecular fluorescence complementation, and so on); and viii) curated genetic associations identified by epistatic analysis and real-time RT-PCR from previous studies and detected from previous gene expression studies as TF-target relationships in transgenic plants where TF were knockout or overexpressed. All the interactions in Arabidopsis were mapped into the ones in rice by identifying the rice orthologs of Arabidopsis genes based on Arabidopsis-rice orthology obtained from InParanoid (Berglund et al. 2008) and the Rice Genome Annotation project (Ouyang et al. 2007).

\section{An interaction network-based method to identify potential $\mathrm{RSV}$ resistance factors.}

We identified the potential RSV resistance factors by the interaction network-based method previously reported (Junker et al. 2006; Kim et al. 2011). For each DEG, we first counted the number of DEG interactors using the interactome described above. Second, we estimated an empirical distribution of the null hypothesis of the number of DEG interactors (i.e., the number of DEG interactors is zero) as the one of the numbers of interactors for all genes in the whole genome. Using the distribution, for each DEG, we then computed the significance (FDR) of its number of DEG interactors using Storey's method. A DEG with a small FDR indicates that it interacts with a large number of DEG. To identify resistance factors which can modulate the activities of the DEG interactors, we focused on the DEG having regulatory functions (e.g., TF, kinases, and various regulators based on GO annotations). Among these DEG, we selected potential host resistance factors as those with FDR $<0.05$. Furthermore, assuming that upregulated genes after RSV infection are more likely to affect resistance-associated processes, we focused on the upregulated genes among the selected potential host resistance factors.

\section{Quantitative real-time RT-PCR analysis of the selected host resistance factors.}

The mRNAs were prepared independently using different seedlings from those used for microarray experiments. However, they were isolated from RSV-infected and RSV-uninfected seedlings of NIL22 and NIL37 lines grown under the same conditions. cDNAs were synthesized from $1.5 \mu \mathrm{g}$ of the mRNAs using a ImProm-II first-strand synthesis system (Promega Corp., Madison, WI, U.S.A.) with an oligo(dT)18 primer. The realtime RT-PCR (SYBR Green PCR Master Mix; Applied Biosystems, Foster City, CA, U.S.A.) was performed and analyzed by using an ABI StepOnePlus (Applied Biosystems). The PCR conditions were $95^{\circ} \mathrm{C}$ for $10 \mathrm{~min}$ and 40 cycles of $95^{\circ} \mathrm{C}$ for 15 $\mathrm{s}, 60^{\circ} \mathrm{C}$ for $30 \mathrm{~s}$, and $78^{\circ} \mathrm{C}$ for $40 \mathrm{~s}$. The analysis was repeated three times for each gene using two biological replicates. The measurement was normalized using the expression level of the gene-encoding ubiquitin fusion protein (LOC_Os03g13170). Expression levels of genes examined from two biological replicates are shown in Supplementary Table S4. Primers used in real-time RT-PCR are listed in Supplementary Table S5.

\section{Reconstruction of a network model.}

To reconstruct an RSV resistance network model for the 21 potential RSV resistance factors, we first identified the DEG interactors of the 21 factors that are involved in the same GO terms with the 21 factors using the rice interactome data described above. This initial network was visualized in Cytoscape (Cline et al. 2007). To incorporate current knowledge into the network, we added known resistance factors (Fig. 5) to the initial network. We then removed the DEG nodes that do not contribute to connecting the 21 factors, known resistance factors, and the nodes in their associated processes. We arranged the nodes into the modules, in each of which the nodes with the same GO term are grouped. The module was labeled with the corresponding GO term. The two sets of the factors that could not be grouped into the modules based on the GO terms (five factors within the QTL on chromosome 11 and five factors selected from the interaction network-based method) were separately located in the network (Fig. 5). For different types of genes, the shapes and colors of the node and boundary were distinctively denoted (Fig. 5, caption). Also, the different shapes and colors of the edges were used to indicate types of interaction data.

\section{ACKNOWLEDGMENTS}

We thank K.-H. Jung at Kyung Hee University for thoughtful discussion and thorough reviews. This work was supported by grants from the BioGreen21 Program in Korean RDA (code 20080401-034-041-008-01-00 and 20080401-034-041-008-02-00 and numberPJ008039); Technology Development Program for Agriculture and Forestry in Korean MIFAFF (309017-5); and WCU program in Korean MEST (R31-2008-000-10105-0). J. Choi was a recipient of a Brain Korea 21 fellowship.

\section{LITERATURE CITED}

Afzal, A. J., Wood, A. J., and Lightfoot, D. A. 2008. Plant receptor-like serine threonine kinases: Roles in signaling and plant defense. Mol. Plant-Microbe Interact. 21:507-517.

Agrawal, G. K., Rakwal, R., Jwa, N. S., and Agrawal, V. P. 2001. Signalling molecules and blast pathogen attack activates rice OsPR1a and OsPR1b genes: A model illustrating components participating during defence/stress response. Plant Physiol. Biochem. 39:1095-1103.

Aparicio, F., Thomas, C. L., Lederer, C., Niu, Y., Wang, D., and Maule, A. J. 2005. Virus induction of heat shock protein 70 reflects a general response to protein accumulation in the plant cytosol. Plant Physiol. 138:529-536.

Berglund, A. C., Sjolund, E., Ostlund, G., and Sonnhammer, E. L. 2008. InParanoid 6: Eukaryotic ortholog clusters with inparalogs. Nucleic Acids Res. 36:D263-266.

Bolstad, B. M., Irizarry, R. A., Astrand, M., and Speed, T. P. 2003. A comparison of normalization methods for high density oligonucleotide array data based on variance and bias. Bioinformatics 19:185-193.

Caplan, J. L., Zhu, X., Mamillapalli, P., Marathe, R., Anandalakshmi, R., and Dinesh-Kumar, S. P. 2009. Induced ER chaperones regulate a receptor-like kinase to mediate antiviral innate immune response in plants. Cell Host Microbe 6:457-469.

Chen, J., Li, W. X., Xie, D. X., Peng, J. R., and Ding, S. W. 2004. Viral virulence protein suppresses RNA silencing-mediated defense but upregulates the role of MicroRNA in host gene expression. Plant Cell 16:1302-1313.

Cline, M. S., Smoot, M., Cerami, E., Kuchinsky, A., Landys, N., Workman, C., Christmas, R., Avila-Campilo, I., Creech, M., Gross, B., Hanspers, K., Isserlin, R., Kelley, R., Killcoyne, S., Lotia, S., Maere, S., Morris, J., Ono, K., Pavlovic, V., Pico, A. R., Vailaya, A., Wang, P. L., Adler, A., Conklin, B. R., Hood, L., Kuiper, M., Sander, C., Schmulevich, I., Schwikowski, B., Warner, G. J., Ideker, T., and Bader, G. D. 2007. Integration of biological networks and gene expression data using Cytoscape. Nat. Protoc. 2:2366-2382.

Du, Z., Zhou, X., Ling, Y., Zhang, Z., and Su, Z. 2010. agriGO: A GO analysis toolkit for the agricultural community. Nucleic Acids Res. 38 (Suppl.):W64-70

Duprat, A., Caranta, C., Revers, F., Menand, B., Browning, K. S., and Robaglia, C. 2002. The Arabidopsis eukaryotic initiation factor (iso) $4 \mathrm{E}$ is dispensable for plant growth but required for susceptibility to potyviruses. Plant J. 32:927-934.

Falk, B. W., and Tsai, J. H. 1998. Biology and molecular biology of viruses in the genus Tenuivirus. Annu. Rev. Phytopathol. 36:139-163.

Feng, B., Chen, Y., Zhao, C., Zhao, X., Bai, X., and Du, Y. 2006. Isolation of a novel Ser/Thr protein kinase gene from oligochitosan-induced tobacco and its role in resistance against Tobacco mosaic virus. Plant Physiol. Biochem. 44:596-603.

Fischer, U., and Droge-Laser, W. 2004. Overexpression of NtERF5, a new member of the tobacco ethylene response transcription factor family enhances resistance to Tobacco mosaic virus. Mol. Plant-Microbe Interact. 17:1162-1171.

Gallie, D. R. 2002. The 5'-leader of Tobacco mosaic virus promotes trans- 
lation through enhanced recruitment of eIF4F. Nucleic Acids Res. 30:3401-3411.

Gao, Z., Johansen, E., Eyers, S., Thomas, C. L., Noel Ellis, T. H., and Maule, A. J. 2004. The potyvirus recessive resistance gene, sbm1, identifies a novel role for translation initiation factor eIF4E in cell-to-cell trafficking. Plant J. 40:376-385.

Geisler-Lee, J., O'Toole, N., Ammar, R., Provart, N. J., Millar, A. H., and Geisler, M. 2007. A predicted interactome for Arabidopsis. Plant Physiol. 145:317-329.

Glazebrook, J. 2005. Contrasting mechanisms of defense against biotrophic and necrotrophic pathogens. Annu. Rev. Phytopathol. 43:205-227.

Glick, E., Zrachya, A., Levy, Y., Mett, A., Gidoni, D., Belausov, E., Citovsky, V., and Gafni, Y. 2008. I with host SGS3 is required for suppression of RNA silencing by Tomato yellow leaf curl virus V2 protein. Proc. Natl. Acad. Sci. U.S.A. 105:157-161.

Gutierrez, R. A., Lejay, L. V., Dean, A., Chiaromonte, F., Shasha, D. E., and Coruzzi, G. M. 2007. Qualitative network models and genomewide expression data define carbon/nitrogen-responsive molecular machines in Arabidopsis. Genome Biol. 8:R7.

Gwizdek, C., Ossareh-Nazari, B., Brownawell, A. M., Doglio, A., Bertrand, E., Macara, I. G., and Dargemont, C. 2003. Exportin-5 mediates nuclear export of minihelix-containing RNAs. J. Biol. Chem. 278:5505-5508.

Hafren, A., Hofius, D., Ronnholm, G., Sonnewald, U., and Makinen, K. 2010. HSP70 and its cochaperone CPIP promote potyvirus infection in Nicotiana benthamiana by regulating viral coat protein functions. Plant Cell 22:523-535

Hayano-Saito, Y., Tsuji, T., Fujii, K., Saito, K., Iwasaki, M., and Saito, A. 1998. Localization of the rice stripe disease resistance gene, Stv-b(i), by graphical genotyping and linkage analyses with molecular markers. Theor. Appl. Genet. 96:1044-1049.

Hayano-Saito, Y., Saito, K., Nakamura, S., Kawasaki, S., and Iwasaki, M. 2000. Fine physical mapping of the rice stripe resistance gene locus, Stvb-i. Theor. Appl. Genet. 101:59-63.

Hernandez-Pinzon, I., de Jesus, E., Santiago, N., and Casacuberta, J. M. 2009. The frequent transcriptional readthrough of the tobacco Tnt1 retrotransposon and its possible implications for the control of resistance genes. J. Mol. Evol. 68:269-278.

Hong, B. S., Cho, J. H., Kim, H., Choi, E. J., Rho, S., Kim, J., Kim, J. H., Choi, D. S., Kim, Y. K., Hwang, D., and Gho, Y.S. 2009. Colorectal cancer cell-derived microvesicles are enriched in cell cycle-related mRNAs that promote proliferation of endothelial cells. BMC Genomics 10:556.

Huang, T. S., Wei, T. Y., Laliberte, J. F., and Wang, A. M. 2010. A host RNA helicase-like protein, AtRH8, interacts with the potyviral genomelinked protein, $\mathrm{VPg}$, associates with the virus accumulation complex, and is essential for infection. Plant Physiol. 152:255-266.

Hubert, D. A., Tornero, P., Belkhadir, Y., Krishna, P., Takahashi, A., Shirasu, K., and Dangl, J. L. 2003. Cytosolic HSP90 associates with and modulates the Arabidopsis RPM1 disease resistance protein. EMBO (Eur. Mol. Biol. Organ.) J. 22:5679-5689.

Jiang, L., Qian, D., Zheng, H., Meng, L. Y., Chen, J., Le, W. J., Zhou, T., Zhou, Y. J., Wei, C. H., and Li, Y. 2012. RNA-dependent RNA polymerase 6 of rice (Oryza sativa) plays role in host defense against negative-strand RNA virus, Rice stripe virus. Virus Res. 163:512-519.

Junker, B. H., Koschutzki, D., and Schreiber, F. 2006. Exploration of biological network centralities with CentiBiN. BMC Bioinf. 7:219.

Kasschau, K. D., Xie, Z. X., Allen, E., Llave, C., Chapman, E. J., Krizan, K. A., and Carrington, J. C. 2003. P1/HC-Pro, a viral suppressor of RNA silencing, interferes with Arabidopsis development and miRNA function. Dev. Cell 4:205-217.

Kim, S., You, S., and Hwang, D. 2011. Aminoacyl-tRNA synthetases and tumorigenesis: More than housekeeping. Nat. Rev. Cancer 11:708-718.

Kitajewski, J., Schneider, R. J., Safer, B., and Shenk, T. 1986. An adenovirus mutant unable to express VAI RNA displays different growth responses and sensitivity to interferon in various host cell lines. Mol. Cell Biol. 6:4493-4498.

Lee, H. J., Suk, J. E., Patrick, C., Bae, E. J., Cho, J. H., Rho, S., Hwang, D., Masliah, E., and Lee, S. J. 2010a. Direct transfer of alpha-synuclein from neuron to astroglia causes inflammatory responses in synucleinopathies. J. Biol. Chem. 285:9262-9272.

Lee, I., Ambaru, B., Thakkar, P., Marcotte, E. M., and Rhee, S. Y. 2010 b. Rational association of genes with traits using a genome-scale gene network for Arabidopsis thaliana. Nat. Biotechnol. 28:149-156.

Lellis, A. D., Kasschau, K. D., Whitham, S. A., and Carrington, J. C. 2002. Loss-of-susceptibility mutants of Arabidopsis thaliana reveal an essential role for eIF(iso)4E during potyvirus infection. Curr. Biol. 12:10461051.

Li, D., Liu, H., Zhang, H., Wang, X., and Song, F. 2008. OsBIRH1, a DEAD-box RNA helicase with functions in modulating defence responses against pathogen infection and oxidative stress. J. Exp. Bot.
59:2133-2146.

Lin, S. S., Henriques, R., Wu, H. W., Niu, Q. W., Yeh, S. D., and Chua, N. H. 2007. Strategies and mechanisms of plant virus resistance. Plant Biotechnol. Rep. 1:125-134.

Linder, P., and Owttrim, G. W. 2009. Plant RNA helicases: Linking aberrant and silencing RNA. Trends Plant Sci. 14:344-352.

Liu, Y. L., Burch-Smith, T., Schiff, M., Feng, S. H., and Dinesh-Kumar, S. P. 2004. Molecular chaperone Hsp90 associates with resistance protein $\mathrm{n}$ and its signaling proteins SGT1 and Rar1 to modulate an innate immune response in plants. J. Biol. Chem. 279:2101-2108.

Lu, R., Malcuit, I., Moffett, P., Ruiz, M. T., Peart, J., Wu, A. J., Rathjen, J. P., Bendahmane, A., Day, L., and Baulcombe, D. C. 2003. High throughput virus-induced gene silencing implicates heat shock protein 90 in plant disease resistance. EMBO (Eur. Mol. Biol. Organ.) J. 22:5690-5699.

Maeda, H., Matsushita, K., Iida, S., and Sunohara, Y. 2006. Characterization of two QTLs controlling resistance to Rice stripe virus detected in a Japanese upland rice line, Kanto 72. Breed. Sci. 56:359-364.

Maule, A. J., Caranta, C., and Boulton, M. I. 2007. Sources of natural resistance to plant viruses: Status and prospects. Mol. Plant Pathol. 8:223-231

Mitsuhara, I., Iwai, T., Seo, S., Yanagawa, Y., Kawahigasi, H., Hirose, S., Ohkawa, Y., and Ohashi, Y. 2008. Characteristic expression of twelve rice PR1 family genes in response to pathogen infection, wounding, and defense-related signal compounds (121/180). Mol. Genet. Genomics 279:415-427.

Mourrain, P., Beclin, C., Elmayan, T., Feuerbach, F., Godon, C., Morel, J. B., Jouette, D., Lacombe, A. M., Nikic, S., Picault, N., Remoue, K., Sanial, M., Vo, T. A., and Vaucheret, H. 2000. Arabidopsis SGS2 and SGS3 genes are required for posttranscriptional gene silencing and natural virus resistance. Cell 101:533-542.

Mukhtar, M. S., Carvunis, A. R., Dreze, M., Epple, P., Steinbrenner, J., Moore, J., Tasan, M., Galli, M., Hao, T., Nishimura, M. T., Pevzner, S. J., Donovan, S. E., Ghamsari, L., Santhanam, B., Romero, V., Poulin, M. M., Gebreab, F., Gutierrez, B. J., Tam, S., Monachello, D., Boxem, M., Harbort, C. J., McDonald, N., Gai, L., Chen, H., He, Y., Vandenhaute, J., Roth, F. P., Hill, D. E., Ecker, J. R., Vidal, M., Beynon, J., Braun, P., and Dangl, J. L. 2011. Independently evolved virulence effectors converge onto hubs in a plant immune system network. Science 333:596-601.

Nieto, C., Morales, M., Orjeda, G., Clepet, C., Monfort, A., Sturbois, B., Puigdomenech, P., Pitrat, M., Caboche, M., Dogimont, C., Garcia-Mas, J., Aranda, M. A., and Bendahmane, A. 2006. An eIF4E allele confers resistance to an uncapped and non-polyadenylated RNA virus in melon Plant J. 48:452-462.

Noueiry, A. O., Chen, J., and Ahlquist, P. 2000. A mutant allele of essential, general translation initiation factor DED1 selectively inhibits translation of a viral mRNA. Proc. Natl. Acad. Sci. U.S.A. 97:12985-12990.

Nurieva, R. I., Chung, Y., Hwang, D., Yang, X. O., Kang, H. S., Ma, L., Wang, Y. H., Watowich, S. S., Jetten, A. M., Tian, Q., and Dong, C. 2008. Generation of T follicular helper cells is mediated by interleukin21 but independent of $\mathrm{T}$ helper 1, 2, or 17 cell lineages. Immunity 29:138-149.

Ouyang, S., Zhu, W., Hamilton, J., Lin, H., Campbell, M., Childs, K., Thibaud-Nissen, F., Malek, R. L., Lee, Y., Zheng, L., Orvis, J., Haas, B., Wortman, J., and Buell, C. R. 2007. The TIGR Rice Genome Annotation Resource: Improvements and new features. Nucleic Acids Res. 35:D883-887.

Pal, A., Chakrabarti, A., and Basak, J. 2007. New motifs within the NBARC domain of $\mathrm{R}$ proteins: Probable mechanisms of integration of geminiviral signatures within the host species of Fabaceae family and implications in conferring disease resistance. J. Theor. Biol. 246:564573.

Peart, J. R., Mestre, P., Lu, R., Malcuit, I., and Baulcombe, D. C. 2005. NRG1, a CC-NB-LRR protein, together with N, a TIR-NB-LRR protein, mediates resistance against Tobacco mosaic virus. Curr. Biol. 15:968-973.

Robaglia, C., and Caranta, C. 2006. Translation initiation factors: A weak link in plant RNA virus infection. Trends Plant Sci. 11:40-45.

Satoh, K., Kondoh, H., Sasaya, T., Shimizu, T., Choi, I. R., Omura, T., and Kikuchi, S. 2010. Selective modification of rice (Oryza sativa) gene expression by Rice stripe virus infection. J. Gen. Virol. 91:294-305.

Shimizu, T., Satoh, K., Kikuchi, S., and Omura, T. 2007. The repression of cell wall- and plastid-related genes and the induction of defense-related genes in rice plants infected with Rice dwarf virus. Mol. Plant-Microbe Interact. 20:247-254.

Shin, R., An, J. M., Park, C. J., Kim, Y. J., Joo, S., Kim, W. T., and Paek, K. H. 2004. Capsicum annuum Tobacco mosaic virus-induced clone 1 expression perturbation alters the plant's response to ethylene and interferes with the redox homeostasis. Plant Physiol. 135:561-573.

Soosaar, J. L., Burch-Smith, T. M., and Dinesh-Kumar, S. P. 2005. Mecha- 
nisms of plant resistance to viruses. Nat. Rev. Microbiol. 3:789-798.

Spear, P. G., Shieh, M. T., Herold, B. C., WuDunn, D., and Koshy, T. I. 1992. Heparan sulfate glycosaminoglycans as primary cell surface receptors for herpes simplex virus. Adv. Exp. Med. Biol. 313:341-353.

Storey, J. D., and Tibshirani, R. 2003. Statistical significance for genomewide studies. Proc. Natl. Acad. Sci. U.S.A. 100:9440-9445.

Summerford, C., and Samulski, R. J. 1998. Membrane-associated heparan sulfate proteoglycan is a receptor for adeno-associated virus type 2 virions. J. Virol. 72:1438-1445.

Takahashi, A., Casais, C., Ichimura, K., and Shirasu, K. 2003. HSP90 interacts with RAR1 and SGT1 and is essential for RPS2-mediated disease resistance in Arabidopsis. Proc. Natl. Acad. Sci. U.S.A. 100:1177711782.

Takahashi, Y., Omura, T., Shohara, K., and Tsuchizaki, T. 1991. Comparison of four serological methods for practical detection of ten viruses of rice in plants and insects. Plant Dis. 75:458-461.

Ulvila, J., Hultmark, D., and Ramet, M. 2010. RNA silencing in the antiviral innate immune defence-role of DEAD-box RNA helicases. Scand. J. Immunol. 71:146-158.

Van Loon, L. C., and Van Strien, E. A. 1999. The families of pathogenesisrelated proteins, their activities, and comparative analysis of PR-1 type proteins. Physiol. Mol. Plant P 55:85-97.

van Ooijen, G., Mayr, G., Kasiem, M. M., Albrecht, M., Cornelissen, B. J., and Takken, F. L. 2008. Structure-function analysis of the NB-ARC domain of plant disease resistance proteins. J. Exp. Bot. 59:1383-1397.

Ventelon-Debout, M., Delalande, F., Brizard, J. P., Diemer, H., Van Dorsselaer, A., and Brugidou, C. 2004. Proteome analysis of cultivarspecific deregulations of Oryza sativa indica and $O$. sativa japonica cellular suspensions undergoing Rice yellow mottle virus infection. Proteomics 4:216-225.

Vidal, M., Braun, P., Carvunis, A. R., Charloteaux, B., Dreze, M., Ecker, J. R., Hill, D. E., Roth, F. P., Vidal, M., Galli, M., Balumuri, P., Bautista, V., Chesnut, J. D., Kim, R. C., de los Reyes, C., Gilles, P., Kim, C. J., Matrubutham, U., Mirchandani, J., Olivares, E., Patnaik, S., Quan, R., Ramaswamy, G., Shinn, P., Swamilingiah, G. M., Wu, S., Ecker, J. R., Dreze, M., Byrdsong, D., Dricot, A., Duarte, M., Gebreab, F., Gutierrez, B. J., MacWilliams, A., Monachello, D., Mukhtar, M. S., Poulin, M. M., Reichert, P., Romero, V., Tam, S., Waaijers, S., Weiner, E. M., Vidal, M., Hill, D. E., Braun, P., Galli, M., Carvunis, A. R., Cusick, M. E., Dreze, M., Romero, V., Roth, F. P., Tasan, M., Yazaki, J., Braun, P., Ecker, J. R., Carvunis, A. R., Ahn, Y. Y., Barabasi, A. L., Charloteaux, B., Chen, H. M., Cusick, M. E., Dangl, J. L., Dreze, M., Ecker, J. R., Fan, C. Y., Gai, L. T., Galli, M., Ghoshal, G., Hao, T., Hill, D. E., Lurin, C., Milenkovic, T., Moore, J., Mukhtar, M. S., Pevzner, S. J., Przulj, N.,
Rabello, S., Rietman, E. A., Rolland, T., Roth, F. P., Santhanam, B., Schmitz, R. J., Spooner, W., Stein, J., Tasan, M., Vandenhaute, J., Ware, D., Braun, P., Braun, P., Carvunis, A. R., Charloteaux, B., Dreze, M., Galli, M., Vidal, M., and Co, A. I. M. 2011. Evidence for network evolution in an Arabidopsis interactome map. Science 333:601-607.

Vogel, J. P., Raab, T. K., Somerville, C. R., and Somerville, S. C. 2004. Mutations in PMR5 result in powdery mildew resistance and altered cell wall composition. Plant J. 40:968-978.

Wang, Y. H., and Warren, J. T., Jr. 2010. Mutations in retrotransposon AtCOPIA4 compromises resistance to Hyaloperonospora parasitica in Arabidopsis thaliana. Genet. Mol. Biol. 33:135-140.

Washio, O., Ezuka, A., Sakurai, Y., and Toriyama, K. 1968. Studies on the breeding of rice varieties resistant to stripe disease. II. Genetic study on resistance to stripe disease in Japanese upland rice. Jpn. J. Breed. 18:96-101.

Whitham, S. A., and Wang, Y. 2004. Roles for host factors in plant viral pathogenicity. Curr. Opin. Plant Biol. 7:365-371.

Whitham, S. A., Quan, S., Chang, H. S., Cooper, B., Estes, B., Zhu, T., Wang, X., and Hou, Y. M. 2003. Diverse RNA viruses elicit the expression of common sets of genes in susceptible Arabidopsis thaliana plants. Plant J. 33:271-283.

Whitham, S. A., Yang, C., and Goodin, M. M. 2006. Global impact: Elucidating plant responses to viral infection. Mol. Plant-Microbe Interact. 19:1207-1215.

Wu, S. J., Zhong, H., Zhou, Y., Zuo, H., Zhou, L. H., Zhu, J. Y., Ji, C. Q. Gu, S. L., Gu, M. H., and Liang, G. H. 2009. Identification of QTLs for the resistance to Rice stripe virus in the indica rice variety Dular. Euphytica 165:557-565.

Wu, X., Zuo, S., Chen, Z., Zhang, Y., Zhu, J., Ma, N., Tang, J., Chu, C., and Pan, X. 2011. Fine mapping of qSTV11 ( TQ ), a major gene conferring resistance to rice stripe disease. Theor. Appl. Genet. 122:915-923.

Xie, Z., Johansen, L. K., Gustafson, A. M., Kasschau, K. D., Lellis, A. D., Zilberman, D., Jacobsen, S. E., and Carrington, J. C. 2004. Genetic and functional diversification of small RNA pathways in plants. PLoS Biol. 2:E104. Published online.

Yafei, C., Yong, Z., Xiaoming, Z., Peng, G., Hailong, A., Yuguang, D., Yingrong, H., Hui, L., and Yuhong, Z. 2009. Functions of oligochitosan induced protein kinase in Tobacco mosaic virus resistance and pathogenesis related proteins in tobacco. Plant Physiol. Biochem. 47:724731.

Zhang, Y. X., Wang, Q., Jiang, L., Liu, L. L., Wang, B. X., Shen, Y. Y., Cheng, X. N., and Wan, J. M. 2011, Fine mapping of qSTV11 (KAS), a major QTL for rice stripe disease resistance. Theor. Appl. Genet 122:1591-1604.

The Acknowledgment section was incorrect in print

The online version was changed on April 16, 2012.

The affiliations for D. Hwang were changed. He was also affiliated with the Department of Chemical Engineering, POSTECH, Pohang, Republic of Korea. The online version was changed on August 2, 2012. 